\title{
Surface analysis and shear bond strength of zirconia on resin cements after non-thermal plasma treatment and/or primer application for metallic alloys
}

\author{
Aljomar José Vechiato-Filho ${ }^{\mathrm{a}, *}$, Adaias Oliveira Matos ${ }^{\mathrm{b}}$, Richard Landers ${ }^{\mathrm{c}}$, Marcelo Coelho Goiato ${ }^{\mathrm{d}}$, \\ Elidiane Cipriano Rangel ${ }^{\mathrm{e}}$, Grace Mendonça De Souza ${ }^{\mathrm{f}}$, \\ Valentim Adelino Ricardo Barão ${ }^{g}$, Daniela Micheline dos Santos ${ }^{\mathrm{h}}$ \\ a Department of Dental Materials and Prosthodontics, Aracatuba Dental School, Univ. Estadual Paulista - UNESP, Aracatuba, Sao Paulo, Brazil \\ ${ }^{\mathrm{b}}$ Department of Prosthodontics and Periodontology, Piracicaba Dental School, University of Campinas (UNICAMP), Piracicaba, Sao Paulo, Brazil \\ c Institute of Physics Gleb Wataghin, University of Campinas (UNICAMP), Cidade Universitária Zeferino Vaz, Barão Geraldo, Campinas, Sao Paulo, Brazil \\ d Department of Dental Materials and Prosthodontics, Aracatuba Dental School, Univ. Estadual Paulista - UNESP, Aracatuba, Sao Paulo, Brazil \\ e Laboratory of Technological Plasmas (LaPTec), Engineering College, Univ. Estadual Paulista - UNESP, Sorocaba, Sao Paulo, Brazil \\ ${ }^{\mathrm{f}}$ Department of Clinical Sciences, Faculty of Dentistry, University of Toronto, Toronto, ON, Canada \\ g Department of Prosthodontics and Periodontology, Piracicaba Dental School, University of Campinas (UNICAMP), Piracicaba, Sao Paulo, Brazil \\ ${ }^{\mathrm{h}}$ Department of Dental Materials and Prosthodontics, Aracatuba Dental School, Univ. Estadual Paulista - UNESP, Aracatuba, Sao Paulo, Brazil
}

\section{A R T I C L E I N F O}

\section{Article history:}

Received 7 July 2016

Received in revised form 6 October 2016

Accepted 8 November 2016

Available online 24 November 2016

\section{Keywords:}

Zirconia

Resin cement

Bond strength

\begin{abstract}
A B S T R A C T
There is no established protocol for bonding zirconia (Y-TZP) with resin cements. Non-thermal plasma (NTP) may be an alternative for the clinical problems related to adhesion. The purpose of the present study was to characterize the surface of Y-TZP exposed to methane $\left(\mathrm{CH}_{4}\right)$ NTP or coated with a layer of primer for metal alloys and the association between the two methods and to evaluate the effect of NTP treatment on bond strength between Y-TZP and two resin cements. A total of 235 Y-TZP discs $(8 \times 2 \mathrm{~mm}$ ) were distributed into five groups: Co (no surface treatment), Pr (primer), NTP (methane plasma), Pr + NTP and NTP + Pr. The effect of the treatment type on the surface free energy, morphology, topography and chemical composition of the Y-TZP discs was investigated. The discs were cemented to composite resin substrates using Panavia F2.0 or RelyX U200. Shear bond strength $(\mathrm{n}=10)$ analyses were performed $(1 \mathrm{~mm} / \mathrm{min})$ before and after thermocycling $\left(5-55^{\circ} \mathrm{C}, 2000 \mathrm{cycles}\right)$ on the bonded specimens. The data were analyzed with one and three-way ANOVAs and Bonferroni tests $(\alpha=$ 0.05). NTP reduced the surface energy and roughness of the Y-TZP discs. SEM-EDS and XPS analyses showed the presence of the organic thin film, which significantly improved the bond strength results when Rely X U200 was used, whereas the primer treatment was more effective with Panavia F2.0. Thermocycling significantly reduced the bond strength results of the NTP and Pr + NTP groups cemented with Rely X U200 and the Pr and NTP + Pr groups cemented with Panavia F2.0. Nonthermal plasma improves the bond strength between Rely X U200 and Y-TZP and also seems to have water-resistant behavior, whereas Panavia F2.0 showed better results when associated with primer.
\end{abstract}

(C) 2016 Elsevier B.V. All rights reserved.

\section{Introduction}

In recent years, dental ceramics with a high crystalline content have been widely used as a core material for fixed restorations because of their high fracture resistance, chemical stability and superior aesthetic properties when compared to other low-fusing ceramic systems [1-7]. However, as the crystalline content increases, the

\footnotetext{
* Corresponding author at: Department of Dental Materials and Prosthodontics, Aracatuba Dental School, Univ. Estadual Paulista - UNESP, Jose Bonifacio, 1153, 16015050 Aracatuba, Sao Paulo, Brazil.

E-mail address: aljomarvechiatoflo@gmail.com (A.J. Vechiato-Filho).
}

glass phase of the ceramic composition is reduced [6]. As a consequence, the etching effect caused by the hydrofluoric acid gel on the glass phase of low-fusing ceramic is not obtained, and this protocol cannot be recommended for yttria-tetragonal zirconia polycrystals (Y-TZP). Although the acid-resistant surface does not seem to be problematic when Y-TZP cores are cemented on ideally anatomic preparations, high bond strength may be required for milled, cast or pressed restorations, which have with misfit of $\approx 100 \mu \mathrm{m}$ and can be considered lesser retentive restorations [1]. Therefore, the development of any approach that could result in a stronger adhesive interface would be useful for those clinical scenarios.

Several approaches have been investigated for enhancing the resin bond to zirconia-based restorations [1,6,8-12]. However, some studies 
have indicated that some of them may create critical flaws in the ceramic surface, leading to their catastrophic failure [6,9,11-13]. These findings may explain the reports of clinical failures [13-16], in spite of the fracture strength values being well above the stresses estimated for the posterior areas of the mouth [13]. Moreover, previous studies have suggested that some primers containing 10methacryloyloxydecyl dihydrogen phosphate (MDP) showed no significant benefit compared to zirconia bonding because this surface treatment does not create a hydrolysis-resistant interface, which results in reductions in bond strength up to $87 \%$ for Panavia cements after 5 months of water storage [6,11].

In addition, nonthermal plasma (NTP) treatments have been considered an alternative solution for clinical problems related to adhesion $[17,18,19]$. In summary, the plasma technique may improve the adhesion by creating new reactive sites onto a substrate surface, which chemically bond to another substrate [17-20]. A previous study demonstrated that thin films created by methane $\left(\mathrm{CH}_{4}\right)$ plasma indeed reduce the contact angle and increase free surface energy of some materials used in biomedical devices [20-22].

Considering that the plasma may be generated containing reactive and energetic species and that the exposure of a material to this environment will inevitably change the properties of the outermost layers, it is speculated that organic films deposited from methane may improve the adhesion between zirconia and resin cements because they would be well connected to the ceramic and possess an affinity to cement. However, to the best of the authors' knowledge, no study has evaluated $\mathrm{CH}_{4}$-based NTP to improve the chemical bonding of Y-TZP to resin cements. Moreover, the association of promising surface treatments has already been investigated by an in vitro investigation, but not for MDP-based primers and plasma methods [8]. Undoubtedly, the durability of this new interface has to be tested through artificial aging $[1,23]$. A thermocycling challenge in an aqueous environment is commonly used to simulate mechanical fatigue in the wet oral environment [1,21,23-27]. Indeed, the hydrolytic stability of the bonding may affect the clinical performance of an indirect restoration because the humidity of the oral environment accelerates the degradation of the adhesive interface. Therefore, thermocycling is considered to provide clinically relevant information [1,21,23-27].

Thus, the aims of this study were: (1) to characterize the surface of Y-TZP discs with or without nonthermal plasma or primer and the association of both based on surface energy, surface roughness, scanning electronic microscopy (SEM), energy dispersive spectroscopy (EDS), and X-ray dispersive spectroscopy (XPS) analyses, and (2) to evaluate the bond strength between Y-TZP-treated surfaces and two resin cements before and after thermocycling. The null hypotheses were that both treatments would not modify the surface characteristics of Y-TZP and affect the bond strength between this ceramic system and tested resin cements.

\section{Materials and methods}

\subsection{Experimental design}

The materials used in the present study are listed in Table 1. A total of 235 discs of Y-TZP ( $8 \mathrm{~mm}$ diameter $\times 2 \mathrm{~mm}$ thickness) were cut and sintered according to manufacturer's instructions. The treatment applied to the Y-TZP disc surfaces were: control (Co, no surface treatment), alloy primer application (Pr), non-thermal plasma treatment (NTP), primer application followed by non-thermal plasma treatment (Pr + NTP), and non-thermal plasma treatment followed by primer application (NTP + Pr). Seven discs per group $(n=7)$ were used for surface energy and surface roughness, SEM-EDS and XPS analyses immediately after surface treatment. Forty discs per group were used for the shear bond strength test, distributed as follows: 20 discs cemented with MDP-based resin cement (Panavia F2.0) and 20 cemented with ester phosphoric methacrylate-based cement (Rely X U200). Ten discs from each group were tested after the bonding procedure, and the remaining 10 were aged by means of thermocycling before being tested for shear bond strength. The flowchart shown in Fig. 1 schematizes the experimental procedures adopted here.

For the sample preparation, the Y-TZP discs were embedded in the center of cylinder cups (SamplKups, Buehler) fabricated by using autopolymerizing acrylic resin (JET, Classico). After its polymerization, the embedded Y-TZP discs were sequentially polished with a preestablished protocol [18]. Discs were then cleaned and degreased with a sequence of alternate ultrasonic bath [18]. The primer was applied onto the Y-TZP surface of the Pr, Pr + NTP and NTP + Pr groups using microbrushes (Microbrush; Microbrush International) for $60 \mathrm{~s}$. Then, the solvent was removed with gentle air jets.

\subsection{NTP treatment}

The Y-TZP discs from the NTP, Pr + NTP and NTP + Pr groups were subjected to NTP treatment. Plasma treatment was performed inside a stainless steel chamber. The system was evacuated to a background pressure of $3.6 \times 10^{-2}$ Torr. Before the $\mathrm{CH}_{4}$ plasma depositions, argon (Ar) gas was admitted into the chamber, and cleaning plasmas were prepared at a radiofrequency of $13.56 \mathrm{MHz}$ ( $70 \mathrm{~W}$ ) and applied in the sample-holder for $600 \mathrm{~s}$ under a constant work pressure of $1.67 \times 10^{-1}$ Torr. The depositing plasma, maintained for $1800 \mathrm{~s}$, were then established by applying a radiofrequency signal $(13.56 \mathrm{MHz}$, $70 \mathrm{~W}$ ) to the top electrode while biasing the sample holder with negative pulses ( $3.12 \mathrm{kV}, 299 \mathrm{~Hz}, 25 \mu \mathrm{s}$ and $0.75 \%$ of duty cycle). Under these conditions, positive ions from the plasma were attracted by the negative potential and implanted on the surface and subsurface region of the samples placed in the biased electrode simultaneously to the film deposition and according to the Plasma Immersion Ion Implantation and Deposition (PIIID) approach. In the first stages of the process, ion

Table 1

Characteristics of materials used in the present study.

\begin{tabular}{|c|c|c|}
\hline Materials & Composition & Fabricant \\
\hline Ceramill zi system & $99 \%$ of $\mathrm{Y}_{2} \mathrm{O}_{3} / \mathrm{HFO}_{2} / \mathrm{Al}_{2} \mathrm{O}_{3} / 1 \%$ of other oxides & ARMANGIRRBACH \\
\hline Z100 composite resin & Bis-GMA/TEDGMA/zirconia/silicon & 3M ESPE \\
\hline Rely X U200 (Base Catalyst) & Vitreous powder/silicon/calcium hydroxide/methacrylate phosphatide ester/di methacrylate/initiators & 3M ESPE \\
\hline Panavia F2.0 (Paste A Paste B & BPEDMA/MDP/DMA/silica/barium sulfate/dibenzoylperoxide N,N-Diethanol-p-toluidine/silica sodiumfluoride & Kuraray America \\
\hline Oxyguard II) & $\begin{array}{l}\text { Polyethyleneglycol/glycerine/sodium benzenesulfinate cont. gel 3-Methacryloxypropil trimethoxy silane/MDP/ethanol } \\
\text { phosphoric acid }\end{array}$ & Inc. \\
\hline Alloy primer & MDP, VBATDT, $98.5 \%$ acetone & $\begin{array}{l}\text { Kuraray America } \\
\text { Inc. }\end{array}$ \\
\hline $\mathrm{CH}_{4}, \mathrm{O}_{2}$ and $\mathrm{Ar}$ & Methane, oxygen and argon & White Martins \\
\hline
\end{tabular}

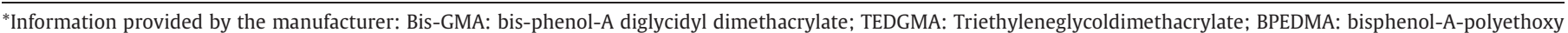
dimethacrylate; DMA: aliphatic dimethacrylate; MDP: 10-methacryloxydecyl dihydrogen phosphate; VBATDT: 6-(4-vinylbenzyl-n-propyl)amino-1,3,5-trizaine-2,4-dithiol. 


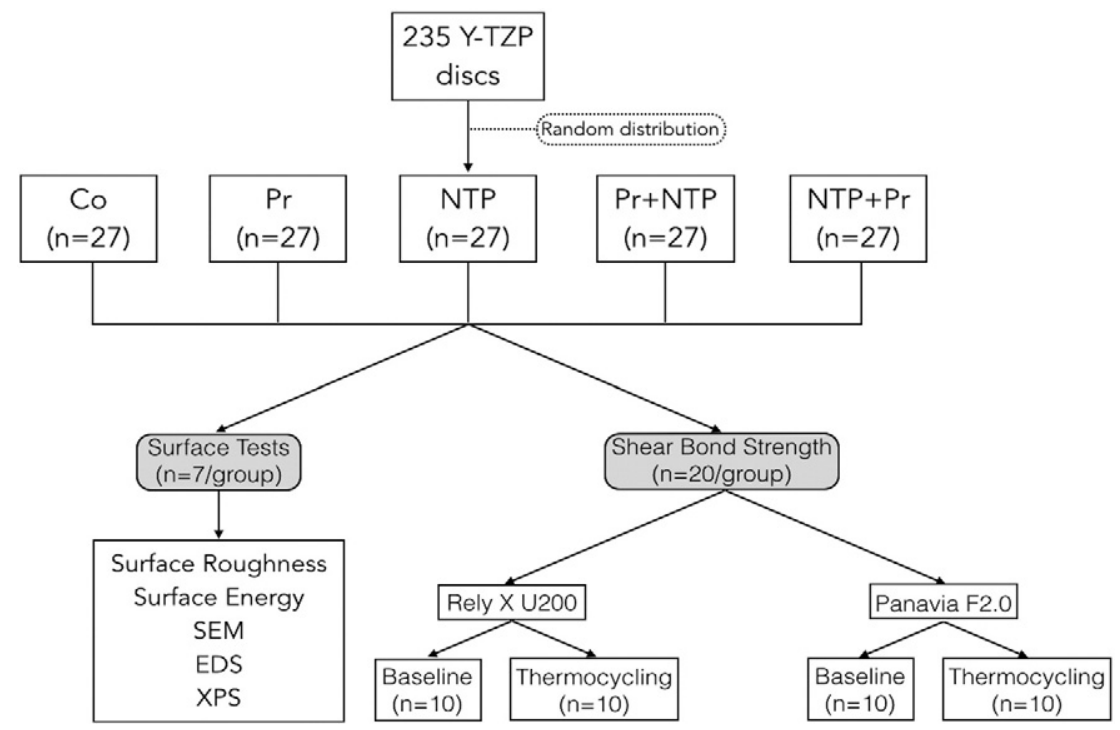

Fig. 1. Flowchart of experimental procedure.

bombardment produced a dilution of the Y-TZP surface with the film constituents, resulting in a diluted film-substrate interface with improved adhesion, mechanical and tribological properties. After the conclusion of the PIID time, the $\mathrm{CH}_{4}$ flow was interrupted while that of $\mathrm{Ar}$ was maintained, generating a non polymerizable ion bombardment procedure named Plasma Immersion Ion Implantation (PIII), which was maintained for $480 \mathrm{~s}$. The discs were removed from the stainless glass chamber at a temperature of $33^{\circ} \mathrm{C}$.

\subsection{Surface energy and roughness}

Three Y-TZP discs from each group were subjected to surface energy analysis with a goniometer (Ramé-Hart 100-00; Ramé-Hart Instrument Co.) using the sessile drop technique. The Owens-Wendt-Rabel-Kaelble method was used to calculate the surface energy by measuring the contact angle between drops of two liquids with different polarities and the Y-TZP disc $[8,9,18,20]$. Surface roughness analysis was performed with a profilometer (Dektak D150; Veeco). Three discs from each experimental group were evaluated. Four parameters of surface roughness were obtained (Ra, Rq, Rz and Rt), following configurations of previous studies $[18,20]$. Then, the average was calculated in nanometers $(\mathrm{nm})[18$, 20].

\subsection{SEM-EDS and XPS analyses}

For SEM analysis, images were captured at $3000 \times$ and $10,000 \times$ magnifications (JSM 610LA; JEOL). Simultaneously, elemental chemical composition analysis was conducted through EDS. The configurations used for EDS analysis followed previous studies [18,20]. The oxide layer of Y-TZP discs was also measured by using X-ray photoelectron spectroscopy (XPS). The spectrometer (Vacuum Science Workshop, VSW HA100) with a hemispherical analyzer was operated in constant transmission mode (line width of $1.6 \mathrm{eV}$ for $\mathrm{Au} 4 \mathrm{f} 7 / 2$ ). Excitation was achieved by using $\mathrm{Al} \mathrm{K \alpha}(1486.6 \mathrm{eV})$ radiation. Measurements were conducted at a pressure $<2 \times 10^{-8}$ mbar. The $\mathrm{C} 1 \mathrm{~s}$ line at $284.6 \mathrm{eV}$ was fixed for surface charging.

\subsection{Shear bond strength test}

For the bonding procedures, composite resin (Z100; 3M-ESPE) cylinders ( $5 \mathrm{~mm}$ in diameter $\times 2.5 \mathrm{~mm}$ in length) were fabricated. For bonding, resin cements were manipulated according to the manufacturer's instructions and placed onto the bottom of the composite resin cylinders with a syringe (Centrix; Nova DFL). Then, composite cylinders with the cement were placed onto Y-TZP discs. Afterwards, a weight of $1000 \mathrm{~g}$ was applied on top of the resin disc for $40 \mathrm{~s}$ with a custom-made device. Then, the excess cement was removed, and the discs were polymerized at 4 different locations for $60 \mathrm{~s}$ each. Afterwards, half of the specimens were immersed in $37^{\circ} \mathrm{C}$ distilled water for $24 \mathrm{~h}$, and the other half were thermocycled (MSCT-3 Plus; Nucci ME) with alternated baths ( $30 \mathrm{~s}$ each) in distilled water between $5{ }^{\circ} \mathrm{C}$ and $55^{\circ} \mathrm{C}$ for $2000 \mathrm{cy}$ cles [20]. The bonded discs were attached to a custom-made holder and tested with a shear bond strength test in a universal testing machine (Instron Model 4400 Universal Testing System, Instron Corporation). A uniaxial tensile force was applied to the adhesive interface through a razor at a constant crosshead speed of $1 \mathrm{~mm} / \mathrm{min}$ until failure of the sample. Shear bond strength results were determined in MPa considering the area of the adhesive interface.

\subsection{Analysis of bonding failure}

The mode of failure between the composite resin cylinder and the YTZP discs was evaluated and quantified using a stereomicroscope (SteREO Discovery V20, Carl Zeiss) at $150 \times$ magnification.

\subsection{Statistical analysis}

Surface energy and surface roughness data were examined by oneway analysis of variance (ANOVA) (factor: surface treatment). Shear bond strength data were analyzed by three-way ANOVA (factor 1: surface treatment; factor 2: resin cement, factor 3: thermocycling). Bonferroni tests were used to compare the mean values among the groups ( $\alpha=0.05$ ) (SPSS version 20.0 - Statistical Package for the Social Sciences, Inc.). Partial Eta Squared was used for effect size measurement. With 10 samples per group in the shear bond strength test, a large size effect was obtained: $\mathrm{F}(6.19)=6.731, P<0.0001, \eta_{\mathrm{p}}^{2}=0.253$.

\section{Results}

Surface treatment significantly affected the surface energy of the YTZP discs ( $F=10.079, P<0.002)$. A significant reduction of surface energy for NTP and Pr + NTP groups was observed when compared to Pr and NTP + Pr groups. Fig. 2 displays the polar and dispersive components of the tested groups. There was a reduction of the polar component for the NTP and Pr + NTP groups, whereas this component was maintained in the Pr group. However, polar and dispersive components 


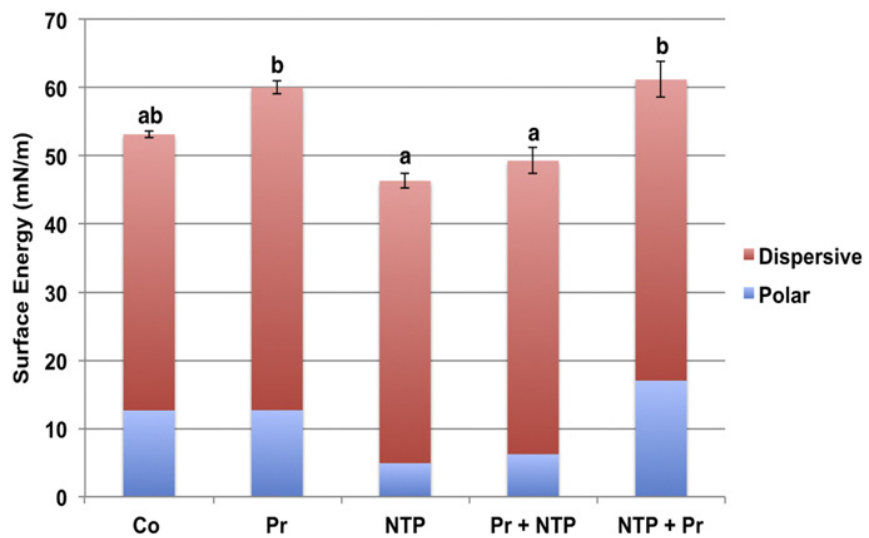

Fig. 2. Main polar and dispersive components of the Co, Pr, NTP, Pr + NTP and NTP $+\operatorname{Pr}$ groups. The different lower case letters represent significant differences $(P<0.001$, Bonferroni test).

were slightly increased in the NTP + Pr group when compared to the control group. Moreover, the one-way ANOVA for surface roughness indicated that surface treatment significantly affected the $\mathrm{Ra}(\mathrm{F}=55.947$, $P<0.001), \operatorname{Rq}(\mathrm{F}=67.384, P<0.001), \operatorname{Rt}(\mathrm{F}=140.430, P<0.001)$ and $\mathrm{Rz}$ $(\mathrm{F}=34.580, P<0.001)$ parameters. The Pr group exhibited the greatest surface roughness, whereas all the other groups were statistically similar to the control (Fig. 3).

SEM-EDS images are displayed in Fig. 4. The Co group had a smooth surface with defects characteristic of the finishing provided to the ceramic surface. This morphology was substantially changed after alloy primer application with an increase in micrometric granular structures dispersed throughout the surface. The NTP treatment also altered the morphology of the pristine material, with the regular matrix being replaced by a fine-grained structure. The Pr + NTP group had an intermediary morphology that did not resemble the film nor the primer, indicating that plasma cleaning ( $\mathrm{Ar}$ ) prior to film deposition may have affected the overall characteristic of the primer surface. In the NTP + Pr group, the surface consistently recovered the aspect of the Pr-containing Y-TZP. Zirconium ( $\mathrm{Zr}$ ), carbon $(\mathrm{C})$ and oxygen $(\mathrm{O})$ were observed in the spectrum of all samples, whereas nitrogen $(\mathrm{N})$ was detected in the Pr, NTP and NTP + Pr groups. For the NTP treated groups, the thin film showed no discontinuity and seemed to be adhered to the Y-TZP surface.

Fig. 5 displays the XPS spectra of tested groups. Only the Co and Pr groups exhibited the $\mathrm{Zr} 3 \mathrm{~d}$ spectrum, where peaks were centered approximately at $182 \mathrm{eV}$ (Fig. 5A). Additionally, for O 1s spectra, peaks can be observed at 530-534 eV for all groups (Fig. 5B). Binding energies at $284-285 \mathrm{eV}$ were seen in the $\mathrm{C} 1 \mathrm{~s}$ spectrum in all groups (Fig. 5C). For the Pr group, the peak centered approximately at $134 \mathrm{eV}$ may be associated with phosphor $(\mathrm{P})$ ions, whereas the peak next to $163 \mathrm{eV}$ may refer to sulfur (S) ions (Fig. 5D-E). Finally, for the N 1s spectra, a peak centered approximately at $399 \mathrm{eV}$ was found for groups subjected to tested surface treatments (Pr, NTP, Pr + NTP and NTP + Pr) (Fig. 5F).

For the shear bond strength, a three-way ANOVA revealed a significant effect of surface treatment $(P<0.001)$, resin cement $(P<0.001)$, thermocycling $(P<0.001)$ and their interactions $(P<0.001)$ (Table 2$)$. Fig. 6 displays the shear bond strength results. The best bond strength results were numerically observed in the NTP group for the Rely X U200, regardless of testing period. For Panavia F2.0, the Pr and NTP + Pr groups showed higher bond strength results at the baseline. The bond strength of samples cemented with Rely X U200 and treated with NTP and Pr + NTP were significantly affected by thermocycling $(P=0.03)$. On the other hand, samples cemented with Panavia F2.0 cement and treated with primer as their outermost surface treatment ( $\mathrm{Pr}$ and NTP + PR) had significant decreases in bond strength after thermocycling $(P<0.001)$.

The failure modes of all the groups are shown in Figs. 7 and 8. An increase in mixed failures after thermocycling was noted for the Pr + NTP and NTP + Pr groups cemented with Rely X U200. There was an overall increase in adhesive failure for all tested groups after thermocycling. No premature failures were observed for either tested cements at baseline. However, 5 premature failures were observed for groups cemented with Rely X U200 and 4 for Panavia F2.0 groups during thermocycling.

\section{Discussion}

This study evaluated the effect of the association between a thin film created by plasma and a primer on the bond strength between zirconia and two different resin cements. In accordance with the results, the null hypotheses of the present study were rejected once the NTP treatment modified the surface characteristics of Y-TZP and improved the shear bond strength of the tested ceramic to both resin cements before and after the thermocycling challenge.

The surface energy is a direct manifestation of intermolecular forces onto a solid surface, which is generally measured by the contact angle of two liquids with different polarities that refers to newer molecular interactions (polar or covalent bonds), such as dipole-dipole interactions, van der Waals forces (Keesom and Debye), hydrogen bonds, and dispersive forces of London $[9,18]$. The deposition of the thin film was responsible for a significant reduction in the surface energy of NTP and $\mathrm{Pr}+$ NTP groups, as well as a reduction in their polar and dispersive

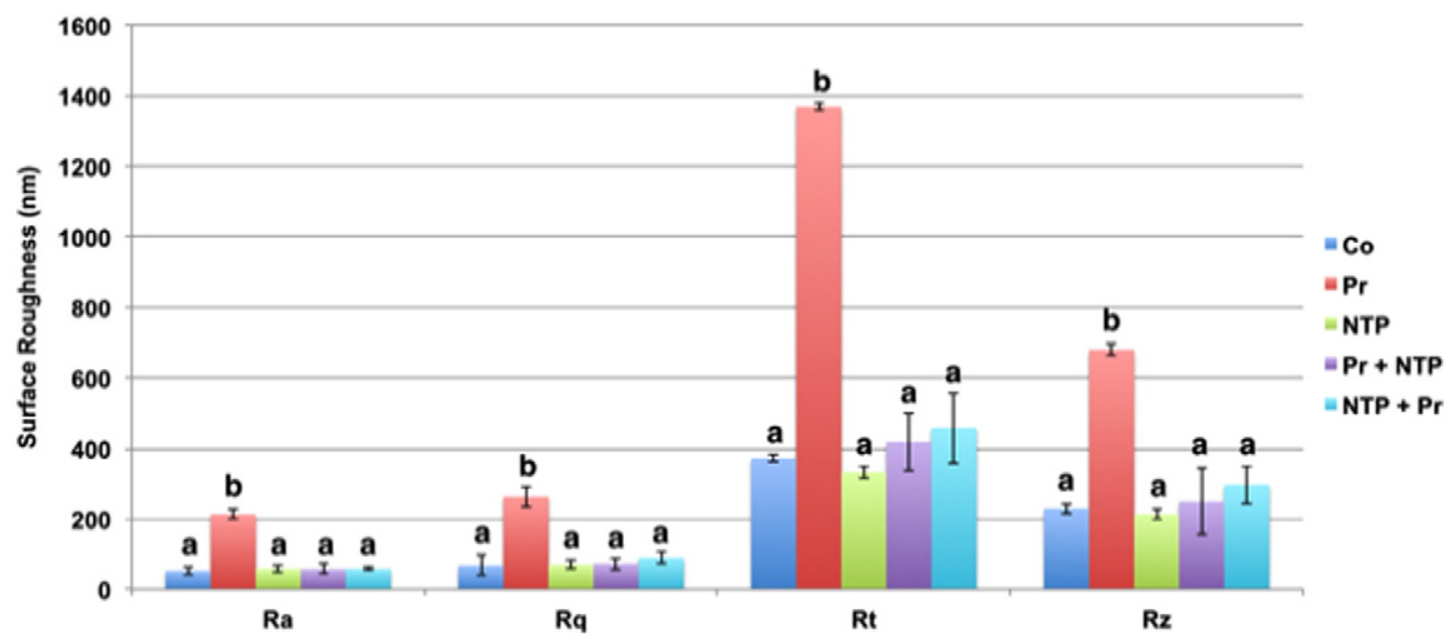

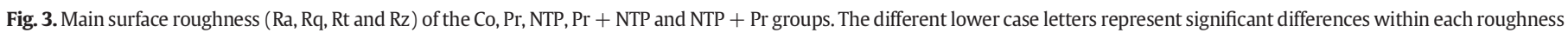
parameter $(P<0.001$, Bonferroni test $)$. 
(A)
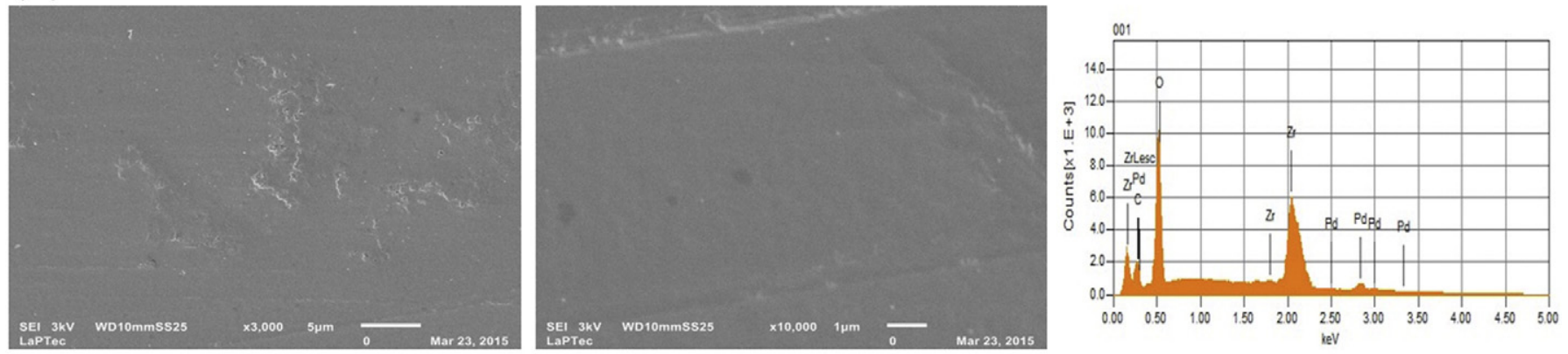

(B)
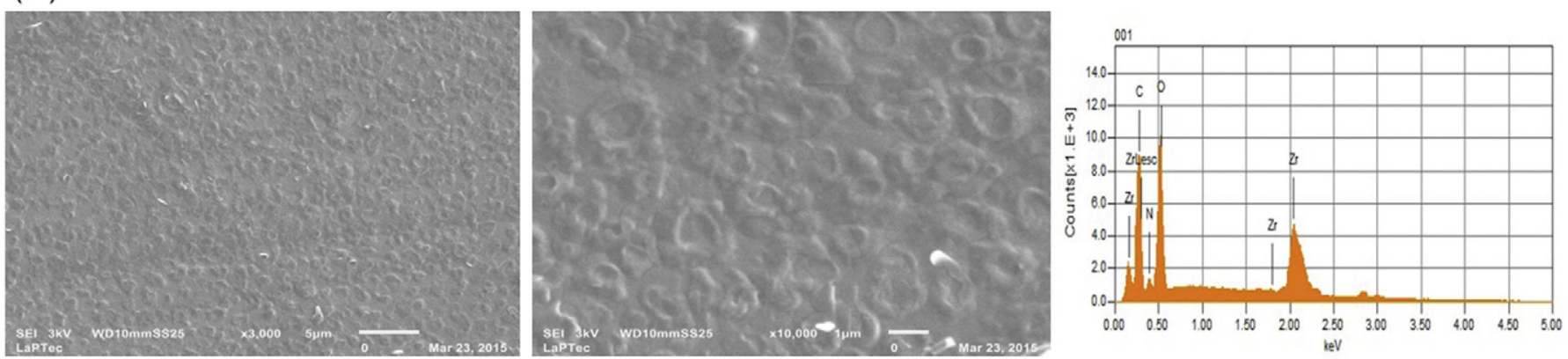

(C)
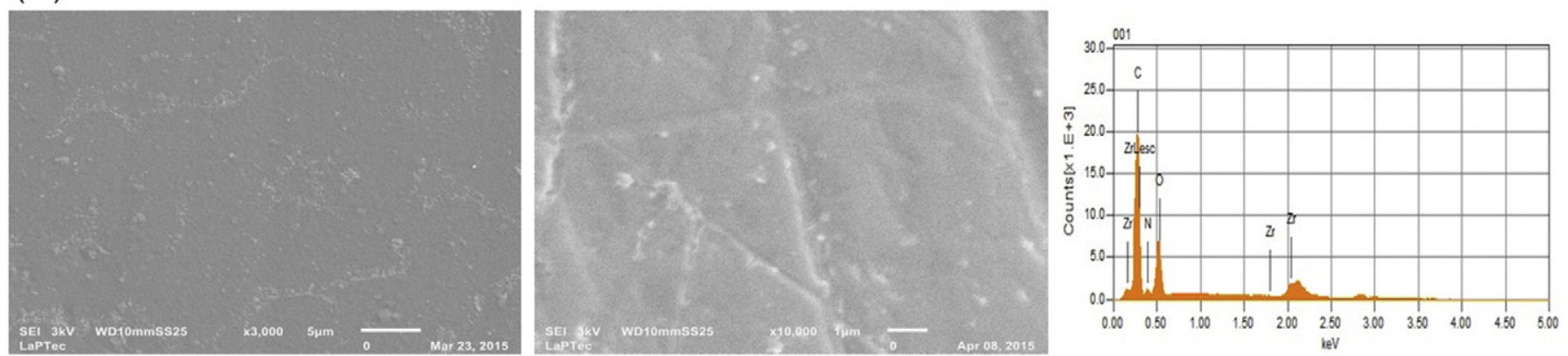

(D)
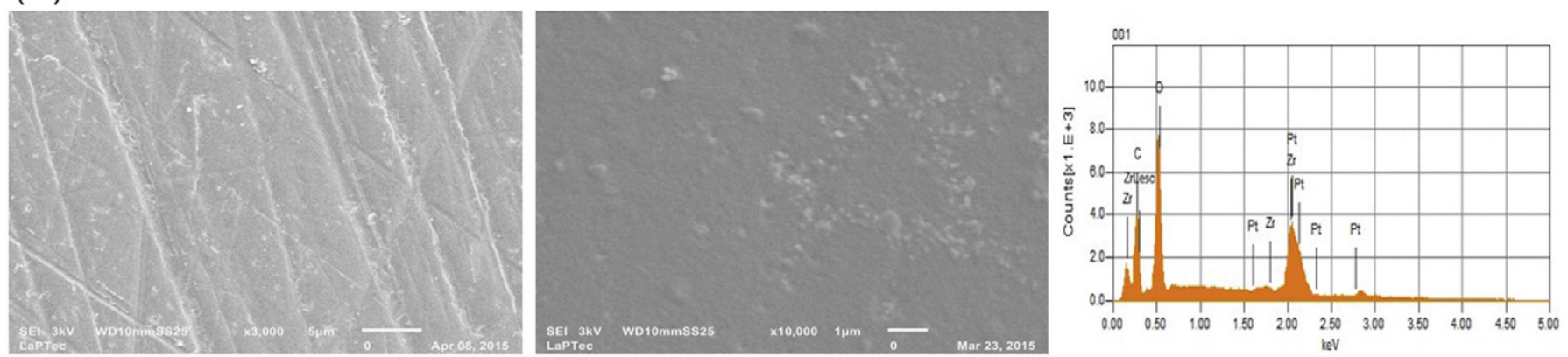

(E)
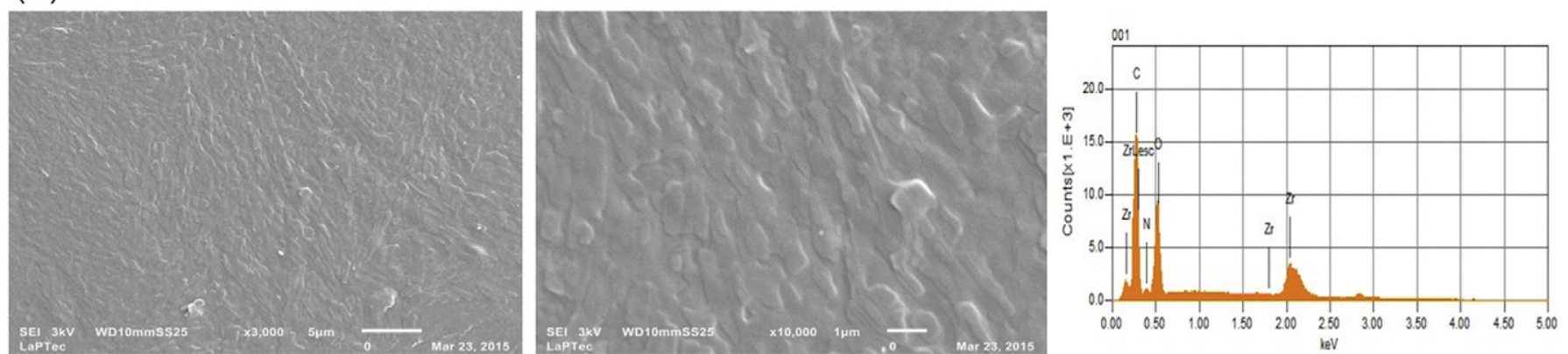

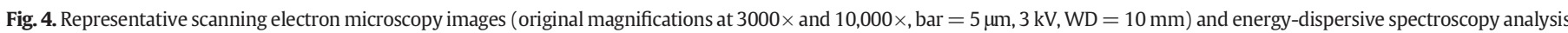
of the Co (A), Pr (B), NTP (C), Pr + NTP (D) and NTP + Pr (E) groups, 

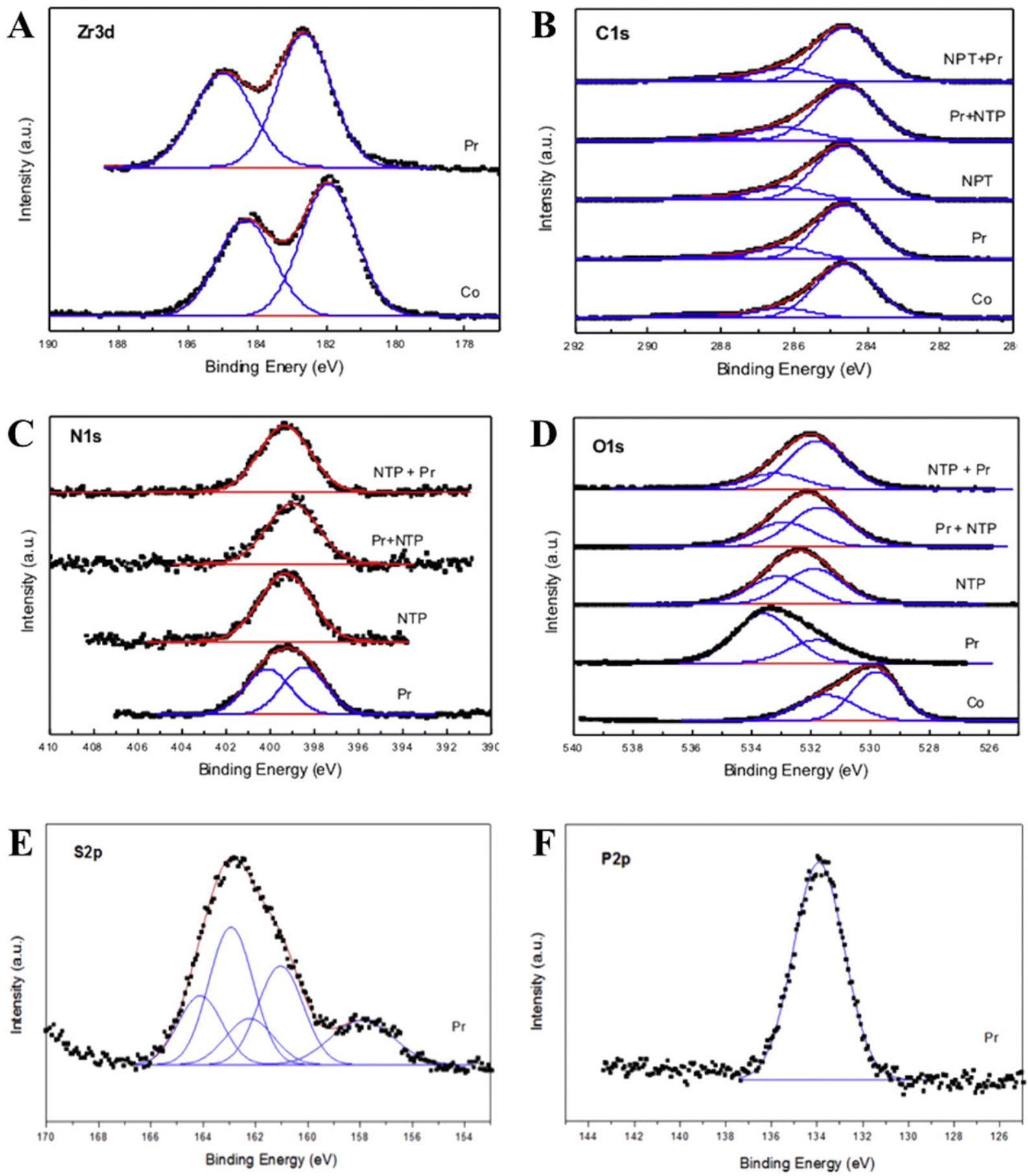

Fig. 5. XPS spectra of (A) Zr 3d, (B) O 1s, (C) C 1s, (D) P 2p, (E) S 2p and (F) N 1s spectra for the experimental groups.

Table 2

Results of three-way analysis of variance (ANOVA) for shear bond strength.

\begin{tabular}{llllll}
\hline Source & Sum of squares & df & Mean square & F & $P$ \\
\hline Surface $(\mathrm{S})$ & 180.361 & 4 & 45.090 & 13.534 & $0.001^{*}$ \\
Cement $(\mathrm{C})$ & 46.918 & 1 & 46.918 & 14.082 & $0.001^{*}$ \\
Thermocycling (T) & 130.739 & 1 & 130.739 & 39.241 & $0.001^{*}$ \\
$\mathrm{~S} \times \mathrm{C}$ & 127.976 & 4 & 31.994 & 9.603 & $0.001^{*}$ \\
$\mathrm{~S} \times \mathrm{T}$ & 74.697 & 4 & 18.674 & 5.605 & $0.001^{*}$ \\
$\mathrm{C} \times \mathrm{T}$ & 16.771 & 1 & 16.771 & 5.034 & $0.026^{*}$ \\
$\mathrm{~S} \times \mathrm{C} \times \mathrm{T}$ & 181.187 & 4 & 45.297 & 13.596 & $0.001^{*}$ \\
Error & 566.393 & 170 & 3.332 & & \\
Total & 7314.767 & 190 & & & \\
Corrected total & 1348.637 & 189 & & & \\
\hline
\end{tabular}

* $P<0.05$ denotes statistically significant difference. components when compared to groups that had the alloy primer as the outermost surface (Pr and NTP + Pr groups) [20]. These findings might be explained by the composition of the thin film. XPS analysis showed that the coating created by the NTP treatment is mainly composed of a tridimensional network of $\mathrm{C}$ atoms (peaks centered approximately at $284 \mathrm{eV} / \mathrm{C}$ 1s spectrum), which does not have chemical affinity to polar components $[19,20]$. This hypothesis can be explained by the ionic bombing during the film deposition. Such method provides sufficient energy to remove the $\mathrm{H}$ ions from $\mathrm{C}$ compounds, which will interact one another and result in a network of amorphous carbons. However, the presence of polar components is observed in all groups. For NTP and $\mathrm{Pr}+$ NTP groups, there is a non-intentional incorporation o $\mathrm{N}$ and $\mathrm{O}$ atoms that can be absorbed from the residual atmosphere into the 


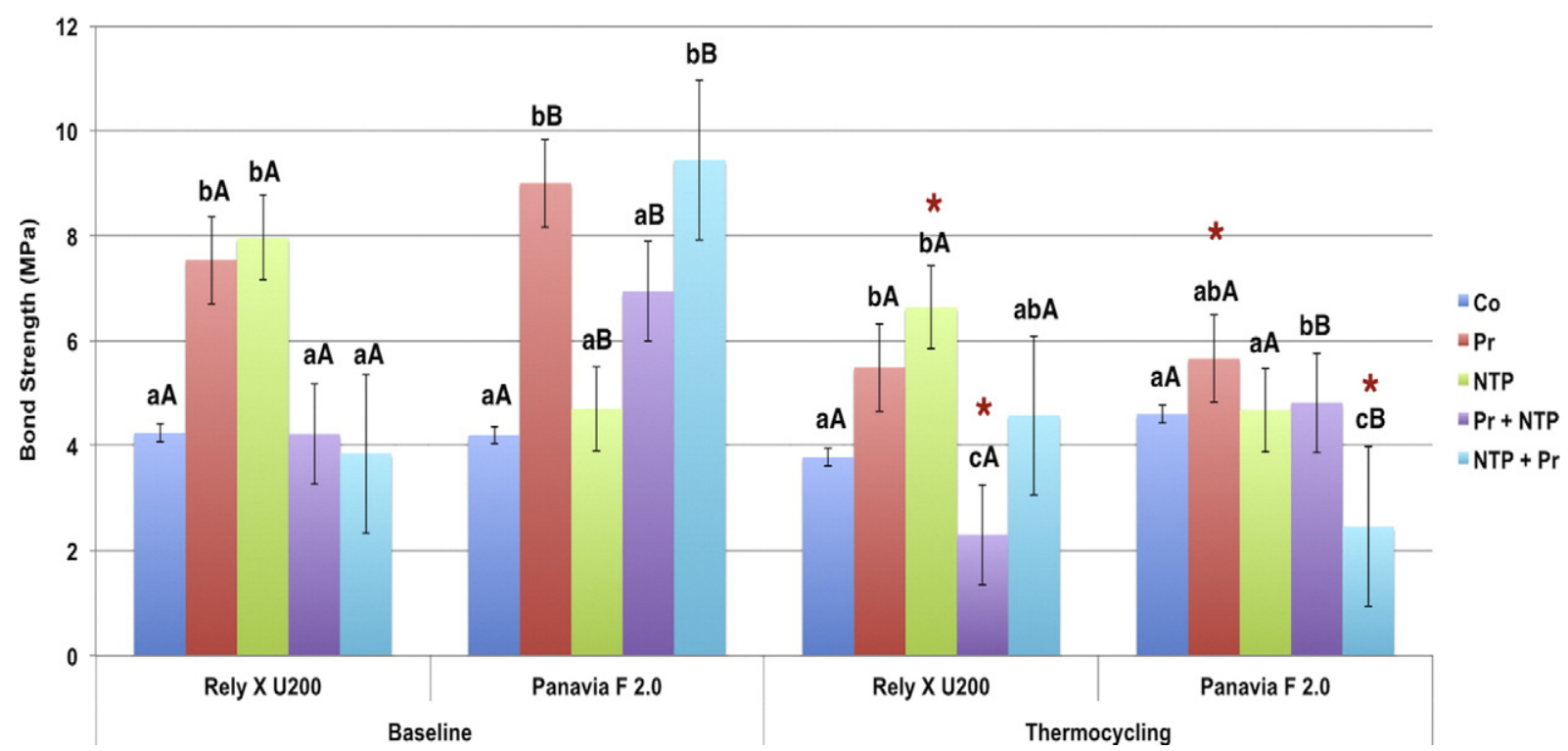

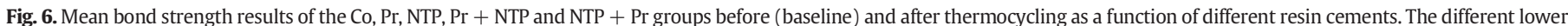

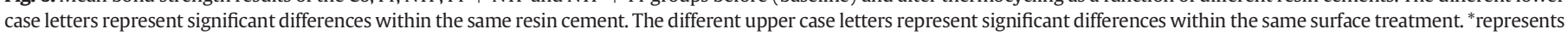
significant differences between periods.

reactor due to unsaturated $\mathrm{C}$ bonds $(\mathrm{C}=\mathrm{C}$ and $\mathrm{C} \equiv \mathrm{C})$ or the reticulation of the network (pending bonds), creating a low number of $C-O, C=0$, $\mathrm{C}-\mathrm{N}, \mathrm{O}-\mathrm{H}$ interactions and a surface that has a low affinity to polar components. Nitrogen and oxygen incorporated in Y-TZP discs of Pr and NTP + Pr groups may result in a more hydrophilic surface because such molecules are in a higher quantity once the Alloy Primer has MDP and VBATDT (6-(4-vinylbenzyl-n-propyl)amino-1,3,5-trizaine-2,4dithiol) molecules in its composition [9]. The incorporation of nitrogen and oxygen to the discs from Pr, NTP, Pr + NTP and NTP + Pr groups is verified by the component centered next to $288 \mathrm{eV}$ that can result from the overlapping nitrogen bonded to $\mathrm{sp}^{3}$ hybridized carbon and peaks at 530-534 eV, respectively. Besides, peaks can be observed at $530-534 \mathrm{eV}$ for all groups that probably correspond to $\mathrm{Zr}-\mathrm{O}$ bonds and $\mathrm{O}^{2-}$ ions bonded to $\mathrm{Al}^{+3}$ ions to form $\mathrm{Al}_{2} \mathrm{O}_{3}$, which can also be attributed to molecules derived from the Y-TZP native layer. Finally, the $\mathrm{Zr} 3 \mathrm{~d}$ spectrum showed peaks that were related to $\mathrm{Zr}-\mathrm{O}$ bonds in $\mathrm{ZrO}_{2}$ and, interestingly, the XPS spectra did not show such binding energies for any groups subjected to NTP treatment, suggesting that the thin film completely covered the $\mathrm{Zr}$ surface.

In addition, the SEM images demonstrated that the NTP treatment applied in the present study showed no topographical alterations to the Y-TZP surface. As reported in a previous study [20], thin films created from $\mathrm{CH}_{4}$ fragmented molecules were deposited at low temperatures [29]. SEM images revealed that NTP treatment did not induce critical defects (Fig. 4). Moreover, no relevant alterations were observed with regard to the surface roughness in the NTP groups compared to the other tested groups, except for the Pr group, in which a layer was created after primer drying. Therefore, misfit of NTP-treated Y-TZP-based restorations would not be observed in a clinical scenario. Thus, it can be assumed that this experimental treatment would be feasible for dental offices because it would not induce subcritical crack growth of the YTZP surface.

The bond strength results can be summarized in two main observations: (1) the thin film deposition had a positive effect on bond strength

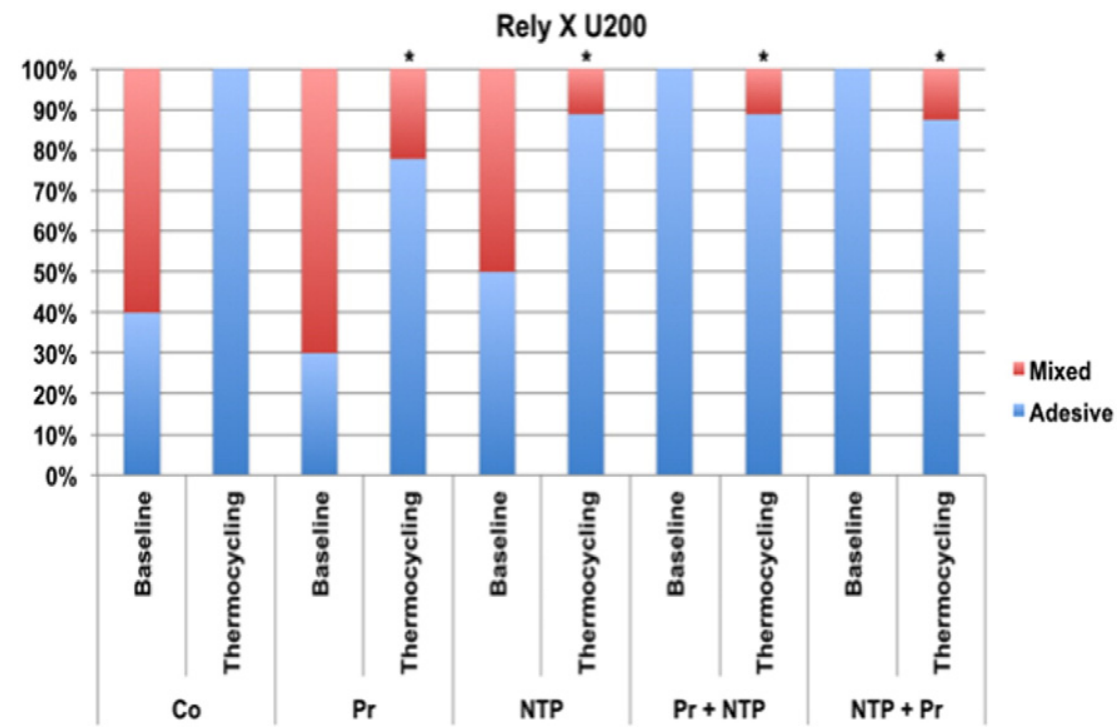

Fig. 7. Incidence of adhesive failure mode for all the samples submitted to the shear bond strength test for Rely X U200. *represents premature failure. 


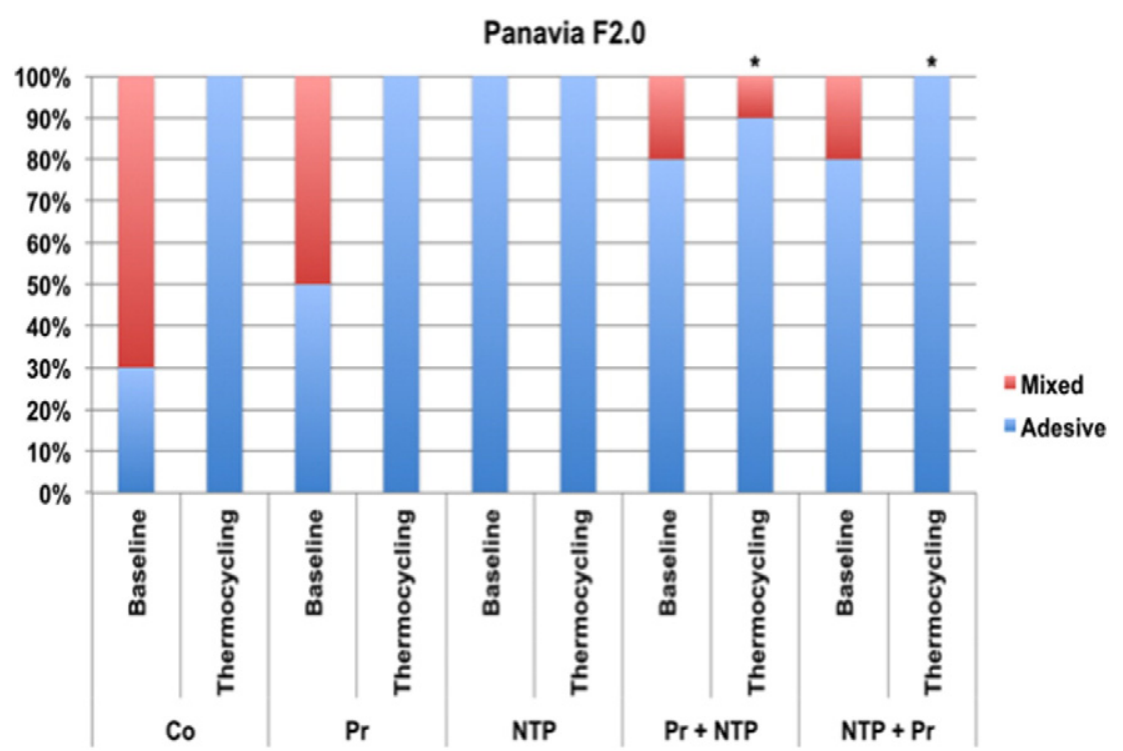

Fig. 8. Incidence of adhesive failure mode for all the samples submitted to the shear bond strength test for Panavia F2.0. *represents premature failure.

results when Rely X U200 cement was used, and (2) the alloy primer was more effective with Panavia F2.0. These findings suggest that there is a poor chemical affinity between thin films from $\mathrm{CH}_{4}$ and Panavia F2.0, which might explain why the artificial aging affected the NTP groups more for this cement than the same groups cemented with Rely XU200. XPS and EDS images showed that the thin film is composed of $\mathrm{C}-\mathrm{C}, \mathrm{C}-\mathrm{O}$ and $\mathrm{C}-\mathrm{N}$ bonds. It is possible that the inner surface of the thin film, which was in contact with the ceramic material, was composed mainly of $\mathrm{C}-\mathrm{O}$ bonds, whereas its external surface (closer to the resin cement) had mostly $\mathrm{C}-\mathrm{C}$ bonds. As a consequence, the effective bond between the acidic groups (phosphoric acid) from MDP did not occur because such molecules might have greater preference for oxides $(\mathrm{ZrO})$ than $\mathrm{C}-\mathrm{C}$ chains $[6,11]$. However, when an alloy primer was applied, MDP from Panavia F2.0 could bond to thiol groups (-SH) from the VBATDT monomer [6,11]. Some studies have reported the presence of carbon (C) and sulfur (S) over the Y-TZP surface after alloy primer application, which may explain this hypothesis [6,11].

This might also explain the better bond strength results of the NTP + Pr group when compared to the NTP group cemented with Panavia F2.0 once the thin film was under the primer layer. Therefore, it did not mask the effect of the outermost surface treatment. This works differently with the Rely X U200. Several studies reported that the ester phosphoric methacrylate from Rely X U200 had chemical affinity for the $\mathrm{C}-\mathrm{C}$ bonds, which interacted directly with the $\mathrm{C}-\mathrm{C}$ bonds of the thin film through secondary chemical interactions $[18,20]$, resulting in an initially high bond strength that was also maintained after aging. Additionally, the presence of $\mathrm{N}$ elements is most likely due to the adsorption from the residual atmosphere $[20,28,29]$. Moreover, differences in chemical affinity might also explain the degradation of the bond at the cement/ceramic interface, which was demonstrated by the incidence of adhesive failures at both periods (Figs. 7 and 8).

With regard to the artificial aging, few significant differences were observed. Surprisingly, the best surface treatments for each tested cement (NTP for Rely X U200 and Pr for Panavia F2.0) and the two groups with combined treatments ( $\mathrm{Pr}+$ NTP with Rely X U200 and NTP + Pr with Panavia F2.0) were significantly affected by thermocycling. However, the NTP group with Rely X U200 showed the highest bond strength results when compared to all tested groups after aging. Thermocycling is an approach used in several in vitro studies to evaluate the hydrolytic degradation of the resin-ceramic bond [1,21,23-27]. Thus, the results presented by $\mathrm{CH}_{4}$ thin film-treated surfaces indicate that this treatment has great potential to fulfill the need for a standard treatment for the inner surface of Y-TZP restorations because it induced no surface modification (damage) and seems to be chemically stable overtime.

Similar to other studies [1,2,5], this research used shear bond strength as the test method to evaluate the adhesion between zirconia and other materials because zirconia is a strong material, and it is extremely difficult to be sectioned. However, questions about the shear bond strength test have been raised because traditional shear protocols may underestimate shear stresses occurring in the clinical scenario, and stress distribution within the adherence zone was not uniform, which may result in significant variation in the mode of failure [1]. Fortunately, this approach provided the advantage of not failing cohesively when submitted to shear forces $[5,30]$, which means that the values obtained represent the true bond strength between the adherent surfaces. Furthermore, it has also been demonstrated that microtensile and shear bond strength methods produce similar results when testing ceramics with a high crystalline content $[5,30]$. Other points to be considered in future studies would be the use of longer, and perhaps more clinically similar, aging protocols, such as a chewing simulation and the evaluation of the treatment efficacy when zirconia is bonded with other resin cements [1].

\section{Conclusion}

Within the limitations of this study, it was possible to conclude that: (1) thin films created from $\mathrm{CH}_{4}$ fragmented molecules could be successfully deposited on a Y-TZP surface, (2) such films increase the resin bond strength of Y-TZP to resin cements mainly composed of ester phosphoric methacrylate, and (3) the adhesive interface has water-resistant behavior. In contrast, Panavia F2.0 showed better results when associated with primer as its outermost layer.

\section{Acknowledgement}

The authors would like to thank to FAPESP (process no. 2014/116024) for financial support.

\section{References}

[1] D.M. Qeblawi, M. Campillo-Funollet, C.A. Muñoz, In vitro shear bond strength of two self-adhesive resin cements to zirconia, J. Prosthet. Dent. 113 (2015) 122-127.

[2] P. Sciasci, F.O. Abi-Rached, G.L. Adabo, P. Baldissara, R.G. Fonseca, Effect of surface treatments on the shear bond strength of luting cements to Y-TZP ceramic, J. Prosthet. Dent. 113 (2015) 212-219. 
[3] A. Attia, M. Kern, Long-term resin bonding to zirconia ceramic with a new universal primer, J. Prosthet. Dent. 106 (2011) 319-327.

[4] F. Lehmann, M. Kern, Durability of resin bonding to zirconia ceramic using different primers, J. Adhes. Dent. 11 (2009) 479-483.

[5] P. Magne, M.P. Paranhos, L.H. Burnett Jr., New zirconia primer improves bond strength of resin-based cements, Dent. Mater. 26 (2010) 345-352.

[6] G.M. Dias de Souza, V.P. Thompson, R.R. Braga, Effect of metal primers on microtensile bond strength between zirconia and resin cements, J. Prosthet. Dent. 105 (2011) 296-303.

[7] S. Kitayama, T. Nikaido, R. Takashashi, L. Zhu, M. Ikeda, R.M. Foxton, A. Sadr, J. Tagami, Effect of primer treatment on bonding of resin cements to zirconia ceramic, Dent. Mater. 26 (2010) 426-432.

[8] G.B. Valverde, P.G. Coelho, M.N. Janal, F.C. Lorenzoni, R.M. Carvalho, V.P. Thompson, K.D. Weltemann, N.R. Silva, Surface characterisation and bonding of Y-TZP following non-thermal plasma treatment, J. Dent. 41 (2013) 51-59.

[9] N.R. Silva, P.G. Coelho, G.B. Valverde, K. Becker, R. Ihrke, A. Quade, V.P. Thompson, Surface characterization of Ti and Y-TZP following non-thermal plasma exposure, J. Biomed. Mater. Res. B Appl. Biomater. 99 (2011) 199-206.

[10] J.Y. Thompson, B.R. Stoner, J.R. Piascik, R. Smith, Adhesion/cementation to zirconia and other non-silicate ceramics: where are we now? Dent. Mater. 27 (2011) 71-82.

[11] G.M. de Souza, N.R. Silva, L.A. Paulillo, M.F. De Goes, E.D. Rekow, V.P. Thompson, Bond strength to high-crystalline content zirconia after different surface treatments, J. Biomed. Mater. Res. B. Appl. Biomater. 93 (2010) 318-323.

[12] M. Kern, A. Barloi, B. Yang, Surface conditioning influences zirconia ceramic bonding, J. Dent. Res. 88 (2009) 817-822.

[13] M. Borba, P.F. Cesar, J.A. Griggs, B.A. Della, Step-stress analysis for predicting dental ceramic reliability, Dent. Mater. 29 (2013) 913-918.

[14] S.S. Scherrer, G.D. Quinn, J.B. Quinn, Fractographic failure analysis of a Procera AllCeram crown using stereo and scanning electron microscopy, Dent. Mater. 24 (2008) 1107-1113.

[15] B. Taskonak, J. Yan, J.J. Mecholsky Jr., A. Sertgöz, A. Koçak, Fractographic analyses of zirconia-based fixed partial dentures, Dent. Mater. 24 (2008) 1077-1082.

[16] S.S. Scherrer, J.B. Quinn, G.D. Quinn, J.R. Kelly, Failure analysis of ceramic clinical cases using qualitative fractography, Int. J. Prosthodont. 19 (2006) 185-192.

[17] G.J. Han, S.N. Chunq, B.H. Chun, C.K. Kim, K.H. Oh, B.H. Cho, Effect of the applied power of atmospheric pressure plasma on the adhesion of composite resin to dental ceramic, J. Adhes. Dent. 14 (2012) 461-469.

[18] A.J. Vechiato Filho, D.M. dos Santos, M.C. Goiato, R.A. de Medeiros, A. Moreno, L.R. Bonatto, E.C. Rangel, Surface characterization of lithium disilicate ceramic after nonthermal plasma treatment, J. Prosthet. Dent. 112 (2014) 1156-1163.
[19] N.C. Cruz, E.C. Rangel, G.Z. Gadioli, R.P. Mota, R.Y. Honda, M.A. Algatti, W.H Schreiner, The influence of plasma composition on the properties of plasma treated biomaterials, MRS Proc. 672 (2001) 03.35, http://dx.doi.org/10.1557/PROC-672-O3. 35.

[20] A.J. Vechiato-Filho, I. da Silva Vieira Marques, D.M. Dos Santos, A.O. Matos, E.C. Rangel, N.C. da Cruz, V.A. Barão, Effect of nonthermal plasma treatment on surface chemistry of commercially-pure titanium and shear bond strength to autopolymerizing acrylic resin, Mater. Sci. Eng. C Mater. Biol. Appl. 60 (2016) 37-44.

[21] C.A. Zamperini, L. Carneiro Hde, E.C. Rangel, N.C. Cruz, C.E. Vergani, A.L. Machado, In vitro adhesion of Candida glabrata to denture base acrylic resin modified by glowdischarge plasma treatment, Mycoses 56 (2013) 134-144.

[22] E. Radeva, L. Pramatarova, E. Pecheva, T. Hikov, E. Iacob, L. Vanzetti, R. Dimitrovac, N. Krastevad, T. Spassove, D. Fingarova, Study of organosilicon plasma polymer used in composite layers with biomedical application, AIP Conf. Proc. 21 (2010) 949-954.

[23] V. Ehlers, G. Kampf, E. Stender, B. Willershausen, C.P. Ernst, Effect of thermocycling with or without 1 year of water storage on retentive strengths of luting cements for zirconia crowns, J. Prosthet. Dent. 113 (2015) 609-615.

[24] J.H. Phark, S. Duarte Jr., M. Blatz, A. Sadan, An in vitro evaluation of the long-term resin bond to a new densely sintered high-purity zirconium-oxide ceramic surface, J. Prosthet. Dent. 101 (2009) 29-38.

[25] M. Kern, S.M. Wegner, Bonding to zirconia ceramic: adhesion methods and their durability, Dent. Mater. 14 (1998) 64-71.

[26] M.B. Blatz, A. Sadan, J. Martin, B. Lang, In vitro evaluation of shear bond strengths of resin to densely-sintered high-purity zirconium-oxide ceramic after long-term storage and thermal cycling, J. Prosthet. Dent. 91 (2004) 356-362.

[27] M. Wolfart, F. Lehmann, S. Wolfart, M. Kern, Durability of the resin bond strength to zirconia ceramic after using different surface conditioning methods, Dent. Mater. 23 (2007) 45-50.

[28] S.A. Pihan, T. Tsukruk, R. Förch, Plasma polymerized hexamethyl disiloxane in adhesion applications, Surf. Coat. Technol. 203 (2009) 1856-1862.

[29] X. Ye, L. Wang, Z.T. Tse, G. Tang, G. Song, Effects of high-energy electro-pulsing treatment on microstructure, mechanical properties and corrosion behavior of Ti-6Al-4V alloy, Mater. Sci. Eng. C Mater. Biol. Appl. 49 (2015) 851-860.

[30] L.F. Valandro, M. Ozcan, R. Amaral, A. Vanderlei, M.A. Bottino, Effect of testing methods on the bond strength of resin to zirconia-alumina ceramic: microtensile versus shear test, Dent. Mater. J. 27 (2008) 849-855. 